\title{
Expresión inmunohistoquímica de ki-67 y p53 en tumor odontogénico queratoquístico en comparación con ameloblastoma multiquístico
}

\author{
Immunohistochemical expression of $\mathrm{Ki}-67$ and p53 in \\ odontogenic keratocysts in comparison with ameloblastomas
}

\author{
Maturana Ramírez A ${ }^{1}$, Aitken Saavedra J11, Reyes Rojas $\mathrm{M}^{2}$, Rojas-Alcayaga G
}

\begin{abstract}
RESUMEN
Introducción: Ha sido descrita la inmunodetección de p53 y Ki-67 en el tumor odontogénico queratoquístico (TOQ) y en ameloblastomas mutiquísticos (AM). Sin embargo, hay escasa y contradictoria evidencia respecto de la comparación de estos dos marcadores entre estas neoplasias. Su estudio podría contribuir a comprender las diferencias que presentan en su comportamiento clínico y ser un complemento discriminatorio al momento de definir tratamiento, pronóstico y recidiva. Objetivo: Comparar el recuento de inmunomarcación de p53 y Ki-67 en células epiteliales de TOQ y AM, presentes en el registro de biopsias del Instituto de Referencia de Patología Oral (IREPO), de la Facultad de Odontología de la Universidad de Chile entre los años 2000-2011. Metodología: Estudio observacional de corte transversal. Se estudiaron mediante inmunohistoquímica 8 casos de TOQ y 6 casos de AM con diagnóstico histopatológico según la clasificación de la OMS del 2005. Las muestras se encontraban en bloques de parafina, fijadas en formalina. Resultados: Los datos mostraron una distribución normal en cuanto al número de células positivas para ambos inmunomarcadores. No hubo diferencias estadísticamente significativas en la inmunomarcación de Ki-67 y p53 para TOQ y AM. Sin embargo, en ambas neoplasias, la inmunomarcación de p53 fue mayor respecto a Ki-67, con una diferencia estadisticamente significativa tanto en TOQ ( $p=0.0134)$ como en AM $(\mathrm{p}=0.0079)$. Conclusión: Los resultados sugieren que la inhibición de apoptosis predominó en ambos tumores por sobre la multiplicación celular. Estas diferencias podrían relacionarse con su potencial de crecimiento.
\end{abstract}

Rev. Clin. Periodoncia Implantol. Rehabil. Oral Vol. 7(1); 12-16, 2014.

Palabras clave: Ameloblastoma, tumor odontogénico queratoquístico, p53, Ki-67.

\begin{abstract}
Introduction: p53 and Ki-67 immunodetection have been described in the keratocystic odontogenic tumor (TOQ) and multicystic ameloblastomas (AM). However, there is limited and contradictory evidence regarding the comparison of these two markers between these neoplasias. Their study could help to understand the differences that occur in their clinical behavior and be a complement when defining discriminatory treatment, prognosis and recurrence. Objective: To compare the immunomarking count of p53 and Ki-67 in epithelial cells in AM and TOQ present in the biopsies registered at the Oral Pathology Reference Institute (IREPO), Faculty of Dentistry, University of Chile from 2000 to 2011 . Methods: Cross-sectional observational study. 8 cases of TOQ and 6 cases of AM with histopathological diagnosis according to the WHO classification of 2005 were studied using immunohistochemistry. The samples were formalin-fixed and paraffin-embedded. Results: The data showed a normal distribution in the number of positive cells for both immunomarkers. There were no statistically significant differences in the Immunohistochemical expression of Ki-67 and p53 of TOQ and AM. However in both tumors, the immunohistochemical expression of p53 was higher compared to Ki-67, with a statistically significant difference in TOQ $(p=0.0134)$ and $A M(p=0.0079)$. Conclusion: The results suggest that inhibition of apoptosis in both tumors predominated over cell multiplication. These differences may be related to their growth potential.
\end{abstract}

Rev. Clin. Periodoncia Implantol. Rehabil. Oral Vol. 7(1); 12-16, 2014.

Key words: Ameloblastoma, odontogenic keratocysts, p53, Ki-67.

\section{INTRODUCCIÓN}

En boca podemos distinguir una extensa variedad de lesiones neoplásicas de diverso origen tisular y con distintos grados de agresividad e invasión, entre las que encontramos a los tumores odontogénicos. Estos tumores constituyen un grupo de enfermedades heterogéneas, que van desde proliferaciones hamartomatosas hasta tumores malignos con capacidad de metástasis ${ }^{(1,2)}$. Derivan de células epiteliales, ectomesenquimales o ambas, que participan en el desarrollo dentario. Su etiología y patogenia es desconocida, sin embargo, se han identificado algunas alteraciones moleculares que podrían participar en su desarrollo y progresión. Clínicamente son asintomáticos, pero pueden producir expansión, movilidad dentaria y reabsorción ósea(3).
De particular interés nos resultan el tumor odontogénico queratoquístico (TOQ) y el ameloblastoma multiquístico (AM), pues siendo ambos tumores epiteliales y agresivos, son considerados neoplasias benignas, las que difieren notoriamente en sus pronósticos y tratamientos ${ }^{(3-5)}$. EI TOQ corresponde a una neoplasia benigna intraosea que presenta un comportamiento potencialmente agresivo e infiltrativo y puede penetrar la cortical ósea comprometiendo estructuras adyacentes. La recidiva es frecuente. Puede presentarse solitario o múltiple, este último es característico del síndrome carcinoma nevoide baso celular ${ }^{(5,6)}$. Por otro lado, el ameloblastoma es una neoplasia localmente invasiva, que histológicamente presenta un patrón folicular o plexiforme en un estroma fibroso. Su etiología es desconocida. Es prácticamente asintomático, limitado a un aumento de volumen en la mayoría de los

\footnotetext{
1. Instructor. Cirujano Dentista. Departamento de Patología y Medicina Oral. Facultad de Odontología, Universidad de Chile. Chile.

2. Instructor. Tecnólogo Médico. Departamento de Patología y Medicina Oral. Facultad de Odontología, Universidad de Chile. Chile.

3. Profesor Asociado. Cirujano Dentista. Departamento de Patología y Medicina Oral. Facultad de Odontología, Universidad de Chile. Chile.
} 
casos, pero manteniendo un carácter infiltrante ${ }^{(7,8)}$

Actualmente se considera que la transformación neoplásica es una acumulación de mutaciónes en una gran región del genoma ${ }^{(8)}$. Entre los marcadores mas destacados, asociados a actividad neoplásica, encontramos p53 y Ki-67. El gen p53 es considerado un modulador esencial en la respuesta celular al estrés exógeno y endógeno ${ }^{(9)}$. Su función es impedir la división de células con material genético dañado, inhibiendo la replicación transcripcional e induciendo la apoptosis celular ${ }^{(10)}$. Las alteraciones en la expresión de p53 es un acontecimiento comun en neoplasias humanas ${ }^{(9,10)}$. En condiciones normales, la proteína p53, considerada un supresor de tumores, se expresa en bajos niveles y actúa como un regulador negativo de la división celular. Las células malignas tienen a menudo altos niveles de expresión de p53 y varios autores han sugerido que este fenómeno confiere una ventaja selectiva en la aparición del fenotipo tumoral(8). En carcinomas de cabeza y cuello, la inactivación de p53 se produce en una alta proporción de casos por la mutación del gen $p 53^{(9)}$. La proteína p53 tiene una vida media de 15 a $20 \mathrm{~min}$, por lo que en condiciones normales no puede ser detectada por inmunohistoquímica. Sin embargo, al mutar el gen se produce una proteína defectuosa que al no poder ser degradada, se acumula por aumento de su vida media, permitiendo de esta manera su inmuno detección. Muchos estudios han utilizado la inmunomarcación de p53 como marcador indirecto de mutación, sin embargo, no se ha demostrado una estricta relación entre la expresión inmunohistoquímica y mutación del gen ${ }^{(10)}$. Finlay et al., sugiere que la expresión moderada o débil de p53 puede indicar un crecimiento lento y expansivo de tumores. En estas células se producirían formas mutantes de p53 capaces de unirse a p53 silvestre, formando complejos inactivos ${ }^{(11)}$. Kumamoto et al. encontraron que aberraciones en la vía p53 (p14ARF-MDM2-p53) en la cascada del sistema de control del ciclo celular, se correlacionan con transformación neoplásica ${ }^{(12)}$.

Por otra parte, el anticuerpo monoclonal Ki-67 detecta un antígeno nuclear que se expresa exclusivamente en las células que entran al ciclo celular (fases G1, S, G2 y mitosis) pero no en G0, por lo es útil para evaluar la tasa de proliferación en tumores. Permite evaluar fácil y rápidamente la proporción de células que proliferan en un tumor, ya que determina la fracción de crecimiento de una población celular ${ }^{(9,13-15)}$. Ha demostrado también ser un buen marcador de recidiva de lesiones tumorales ${ }^{(7,10,16)}$ y se ha correlacionado con estadios TNM más avanzado y metástasis ${ }^{(9)}$.

La inmunodetección de de ambas proteínas ha sido asociada principalmente a neoplasias malignas. Considerando que los tumores odontogénicos son neoplasias benignas pero infiltrantes, cabe preguntarse si los mecanismos de crecimiento e infiltración son semejantes a neoplasias malignas en TOQ y AM.

Encontramos en la literatura detección de p53 y Ki-67, tanto en TOQ como en AM, pero comparados con otros tipos de tumores o quistes. Existe escasa y contradictoria evidencia respecto de la comparación de estos dos marcadores entre estas dos neoplasias. Su estudio podría contribuir a comprender las diferencias que presentan en su comportamiento clínico y ser un complemento discriminatorio al momento de definir tratamiento, pronóstico y recidiva.

Finalmente, y dado que ambos marcadores han sido asociados con una función específica, posibles diferencias en su inmunodetección, permitirían contribuir a establecer si el comportamiento clínico tanto de TOQ como de AM está mayormente determinado por la proliferación celular (Ki-67) o por la inhibición de la apoptosis (p53), información que podría utilizarse para mejorar la comprensión de su comportamiento biológico. Esto se puede traducir en el futuro en mejores y mas específicos y efectivos tratamientos lo que impactaría positivamente en la calidad de vida de los sujetos que los presentan.

\section{MATERIAL Y MÉTODO}

Se seleccionaron, a partir de la base de datos del IREPO de la Facultad de Odontología de la Universidad de Chile, entre los años 2000 y 2011, muestras de TOQ y AM incluidos en bloques de parafina con diagnóstico histopatológico confirmado, según la clasificación de la OMS del $2005^{(17)}$. Todas las muestras debían estar indexadas en el registro del IREPO y en caso de TOQ, las biopsias tuvieron que ser tomadas previas al proceso de descompresión. Fueron excluidas las muestras que presentaban una extensa área de inflamación y/o necrosis o que tenían artefactos de técnica quirúrgica y/o de procesamiento histológico.
Se utilizaron 8 biopsias con diagnóstico histopatológico de TOQ y 6 biopsias con diagnóstico histopatológico de AM.

Con los casos ya seleccionados, un patólogo experto (GR) del Departamento de Patología y Medicina Oral de la Facultad de Odontología de la Universidad de Chile, utilizando un microscopio óptico Olympus CX21, observó las placas histológicas, teñidas con hematoxilina-eosina con el objetivo de confirmar el diagnóstico histopatológico de cada caso, aplicar criterios de inclusión y exclusión y seleccionar el bloque más representativo en los casos en que existía más de un bloque de parafina.

Una vez que se obtuvieron los moldes seleccionados, a cada bloque de parafina se le realizaron 2 cortes de $4 \mu \mathrm{m}$ con navajas de corte para tejidos duros y blandos marca Feather, en micrótomo marca E. Leitz Wetzlar. Posteriormente, fueron colocadas en un baño de estirado marca Labline a $38^{\circ} \mathrm{C}$. Cada corte fue recogido en portaobjeto silanizado y secados a $60^{\circ} \mathrm{C}$ por 30 minutos en estufa marca Memmert. Paralelamente, se utilizaron controles positivos de muestras de adenocarcinoma de colon, recomendado para el anticuerpo p53 por el fabricante y con un carcinoma oral, en el que previamente se había demostrado positividad para Ki-67. Se utilizó como control negativo, cortes de las mismas muestras del estudio en las cuales se reemplazó el anticuerpo primario por PBS. Tanto para p53 como para Ki-67, se utilizaron anticuerpos monoclonales de ratón, RTU, con patrón de distribución nuclear.

Una vez realizada la inmunohistoquímica, las láminas teñidas se numeraron según el número de molde correspondiente y se etiquetaron especificando el anticuerpo utilizado. Luego, se buscaron microscópicamente áreas representativas a un aumento de $10 \mathrm{x}$ y posteriormente se fotografiaron a un aumento de 40x. Los campos representativos fueron determinados por áreas con un mayor número de células específicas de cada neoplasia. En los casos en que se contó con muchos segmentos representativos, se seleccionó el que presentó mayor inmunomarcación. De la zona seleccionada, se obtuvo una foto con el programa Micrometrics SE Premiun, y luego se analizó en el software ImageJ, lo que permitió realizar el conteo de células teñidas. Para evaluar la tinción de p53 y Ki-67, se contabilizó sin considerar intensidad, tinción nuclear de las células epiteliales con color café. El porcentaje de tinción nuclear fue evaluado cada 1000 núcleos en cada caso. Las células evaluadas en el caso del TOQ, fueron de la capa basal y parabasal. Se excluyeron zonas de necrosis, inflamación y artefacto.

El diseño de este estudio fue de tipo observacional de corte transversal y el muestreo de tipo no probabilístico por conveniencia Se realizó un análisis exploratorio de los datos mediante estadística descriptiva. Se determinó distribución normal a través de la prueba de Shapiro Wilk. Se utilizó el Test Student para muetras pareadas. Se estimaron diferencias considerando un nivel de significancia del $5 \%$ $(p \leq 0.05)$. Todas las pruebas estadísticas se realizaron mediante el software Stata 11.0.

\section{RESULTADOS}

La muestra estuvo consitiuida por 8 casos de TOQ y 6 casos de $\mathrm{AM}$, lo que representa un $57.14 \%$ y $42.86 \%$, respectivamente.

La inmunomarcación de Ki-67 en TOQ (Figura 1), obtuvo un promedio de 250 células teñidas ( $D S \pm 134.9$ ), mientras que en $A M$ (Figura 2), obtuvo un promedio de 160 (DS \pm 119.1 ) células teñidas.

La inmunomarcación de p53 en TOQ (Figura 3) obtuvo un promedio de 467 (DS \pm 185.1 ) células teñidas, mientras que en $A M$ (Figura 4), obtuvo un promedio de 505 (DS \pm 301.9 ) células teñidas.

El control positivo de adenocarcinoma de colon obtuvo un promedio de 148 (DS \pm 10.78 ) y el control positivo de Ki-67, muestra 10250, obtuvo un promedio de 193 (DS \pm 58.8 ).

El número de células positivas para ambos inmunomarcadores mostró una distribución normal. Como se observa en el Gráfico 1, el porcentaje de inmunomarcación para p53 fue de un 47\% en TOQ y de un $50 \%$ en $\mathrm{AM}$, mientras que para $\mathrm{Ki}-67$ fue de un $25 \%$ en TOQ y de un $16 \%$ en AM. En ambas neoplasias, la inmunomarcación de p53 fue mayor respecto a Ki-67, con una diferencia estadísticamente significativa tanto en TOQ $(p=0.0134)$ como en AM $(p=0.0079)$. La comparación en la inmunomarcación de Ki-67 entre TOQ y AM no mostró diferencias estadísticamente significativas, como tampoco la inmunomarcación de p53 entre AM y TOQ. 


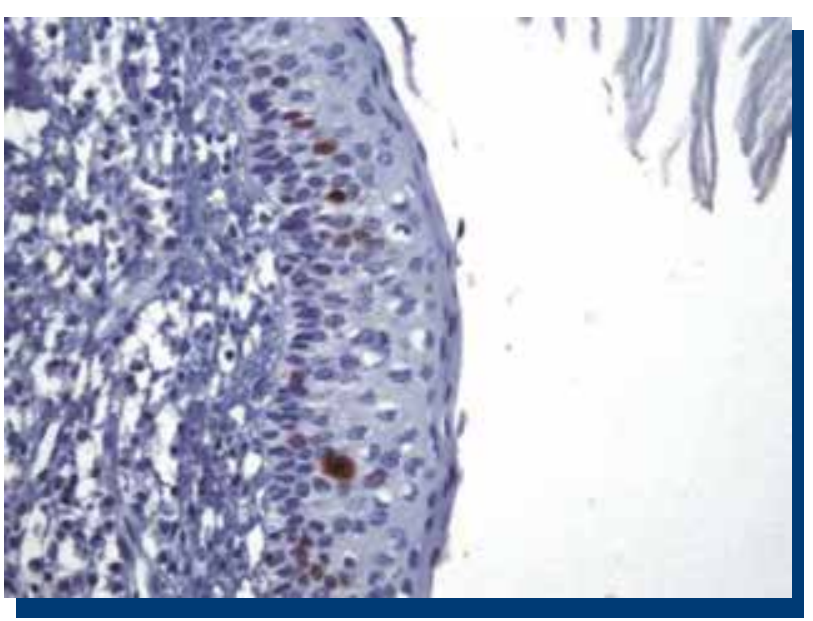

Figura 1. Inmunomarcación positiva para Ki-67 en tumor odontogénico queratoquístico (40x).

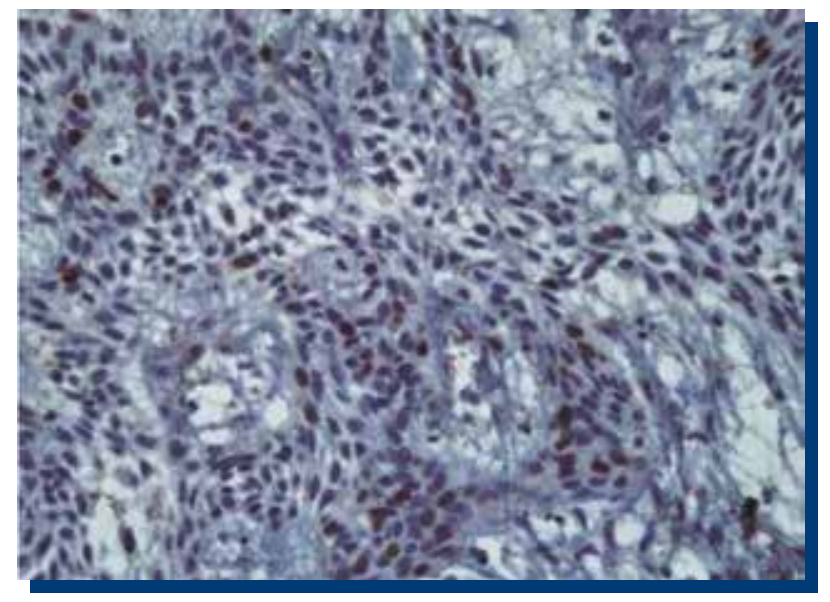

Figura 2. Inmunomarcación positiva para Ki-67 en ameloblastoma multiquístico (40x).

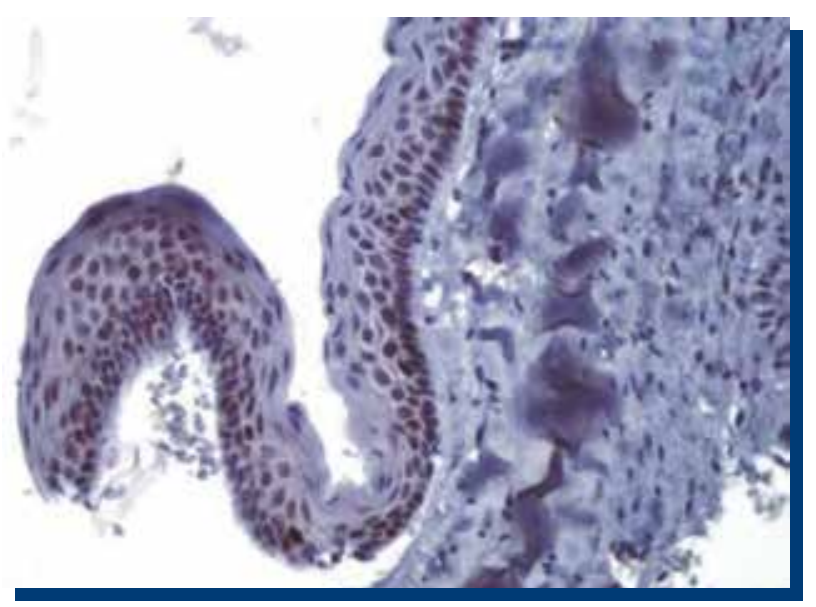

Figura 3. Inmunomarcación positiva para p53 en tumor odontogénico queratoquístico (40x).

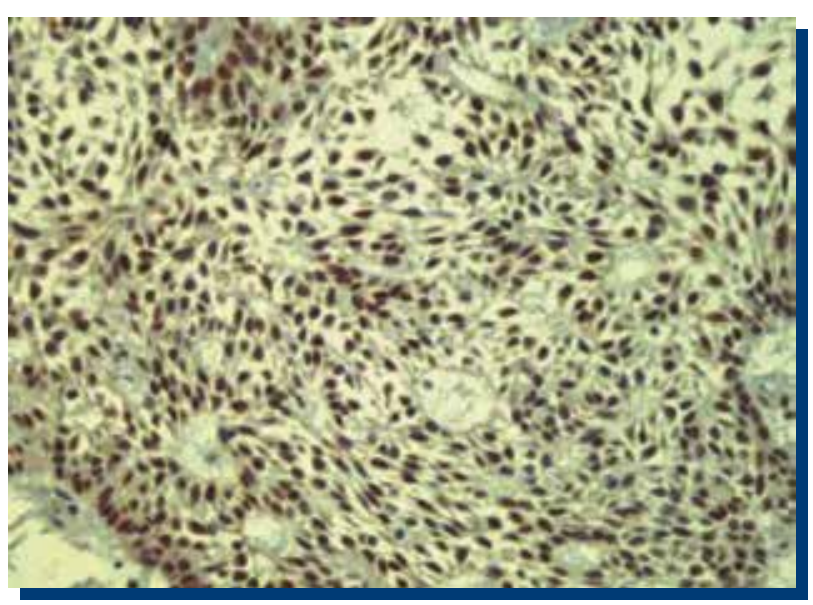

Figura 4. Inmunomarcación positiva para p53 en ameloblastoma multiquístico (40x).

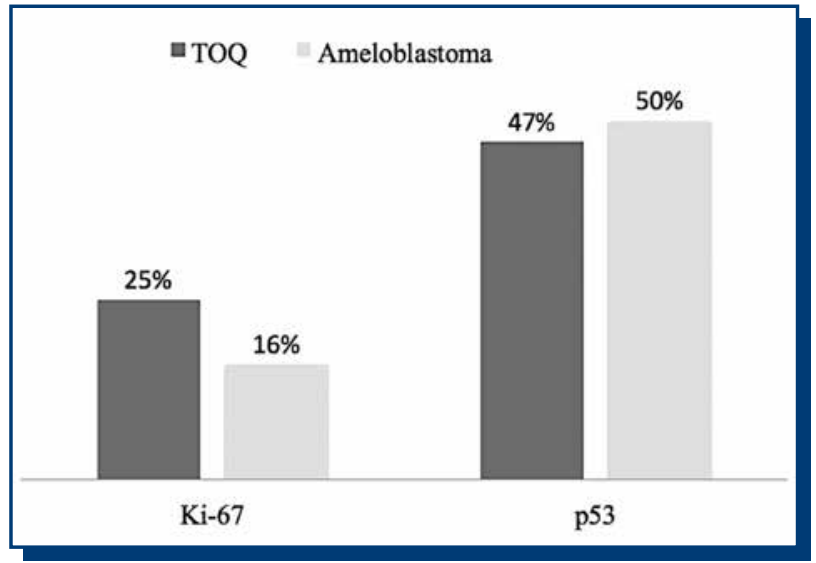

Gráfico 1. Porcentajes de inmunomarcación de Ki-67 y p53 en ameloblastoma y tumor odontogénico queratoquístico.

\section{DISCUSIÓN}

Nuestro objetivo fue comparar el recuento de inmunomarcación de p53 y Ki-67 en TOQ y AM. En ambos tumores la inmunomarcación de p53 fue mayor respecto a Ki-67, con una diferencia estadísticamente significativa. El porcentaje de inmunomarcación para p53 fue de un 47\% en TOQ y de un $50 \%$ en AM, mientras que para Ki- 67 fue de un $25 \%$ en TOQ y de un $16 \%$ en AM.

Respecto del porcentaje de marcación de p53, nuestro estudio no mostró diferencias significativas entre ambas neoplasias. Es más, los datos obtenidos muestran valores similares. De Vicente et al., describen una inmunomarcación de p53 de $30 \%$ en ameloblastomas y de un $50 \%$ en TOQ, y si bien se puede apreciar una diferencia porcentual entre ambas lesiones, al igual que nuestro estudio, no exhiben diferencias significativas ${ }^{(18)}$. En el año 2005, Barboza et al., compararon la inmunomarcación de p53 y antígeno celular de proliferación nuclear (PCNA) en ameloblastoma y en tumor odontogénico adenomatoide (TOA) y dado que el ameloblastoma presentó mayor potencial proliferativo (37.2\% de inmunomarcación de p53), se podría explicar su comportamiento más agresivo e invasor ${ }^{(19)}$. EI PCNA marca positivo en las fases del ciclo celular y en $\mathrm{G} 0$ y se asocia a los procesos de reparación del ADN, por lo que su marcación no está exclusivamente indicando proliferación celular ${ }^{(20)}$. Este marcador, sin embargo, no fue utilizado en nuestra investigación. Salehinejad et al., obtuvieron diferencias significativas al comparar la inmunomarcación de p53 en ameloblastoma $(43.97 \%)$ y en TOA $(6.75 \%)^{(21)}$, mientras que Florescu et al., describieron en ameloblastomas valores cercanos al $50 \%$ de inmunomarcación de $\mathrm{p} 53^{(8)}$. Ambos estudios exhiben resultados similares al nuestro, respecto al porcentaje de marcación de p53 en ameoblastomas.

Piattelli et al., mostraron una mayor inmunomarcación de 
p53 en TOQ comparado con quistes dentigeros y quistes radiculares, lo que podría estar asociado con la presencia de displasia epitelial en $\mathrm{TOQ}^{(22)}$. Shear, el año 2002, concluye que p53, PCNA y Ki-67 presentan una mayor inmunomarcación en TOQ en comparación con otros quistes odontogénicos ${ }^{(23)}$. Slootweg et al., proponen que la sobreexpresión de p53 estaría relacionada con la capacidad proliferativa del TOQ ${ }^{(24)}$, relación también sugerida por González-Moles, debido a que en las muestras evaluadas observó mayor positividad para p53 en zonas con intensa marcación de proliferación evaluadas con Ki-67 y PCNA(25). Li et al., sugiere que la mayor expresión de p53 en TOQ en comparación con otros quistes odontogénicos, no sería el resultado de una mutación del gen p53, sino de la sobreexpresión y/o estabilización de p53 normal relacionada con la proliferación celular(26).

Respecto a Ki-67 en ameloblastomas, se obtuvo una inmunomarcación del $25 \%$ en células epiteliales. En general, la literatura describe porcentajes de proliferación de este marcador que varían desde un $7.8 \%^{(18)}$ hasta un $64.9 \%{ }^{(4)}$. Florescu et al., estudiaron en ameloblastomas la expresión de p53, Bcl-2 y Ki-67 en celulas epiteliales tumorales. La inmunoexpresión de p53 y Bcl-2 se identificó en más del $50 \%$ de las células marcadas ${ }^{(8)}$. Ki-67 en TOQ, presenta en general en la literatura porcentajes mayores a los nuestros, con valores cercanos al $40 \%{ }^{(18)}$. Autores como Mendes et al., compararon la inmunomarcación en TOQ de $\mathrm{p} 53, \mathrm{Ki}-67$ y COX2, sin encontrar diferencias significativas ${ }^{(6)}$. Otros autores como Soluk Tekkeşin et al. y Amaral et al., al comparar TOQ y ameloblastoma, encontraron diferencias significativas en la expresión de Ki-67, siendo, en ambos estudios, mayor inmunomarcación en TOQ que en ameloblastoma ${ }^{(20,27)}$. Estos resultados no se corresponden con los nuestros, donde si bien la marcación de Ki-67 fue mayor en TOQ en comparación con AM, la diferencia no fue significativa. Mendes et al., el 2010, realizaron una revisión bibliográfica con una serie de inmunomarcadores de proliferación y apoptosis evaluados en TOQ. En estos estudios, TOQ presenta una fuerte tinción con marcadores como Ki-67, PCNA y p53, comparado con otros quistes odontogénicos. La expresión de Ki-67 demostró ser superior en el epitelio del TOQ en comparación con quistes del desarrollo e inflamatorios, además de una mayor presencia de Ki-67 en capas suprabasales ${ }^{(5)}$. En un estudio realizado en 2006 por Migaldi M et al., se evaluó proliferación celular mediante Ki-67 y alteraciones en microsatélites en ameloblastomas. Los microsatélites son secuencias cortas repetitivas de DNA, 1-10 pares de bases, que se producen en las regiones no codificantes del ADN. La presencia de alteraciones en microsatélites fue más frecuente en tumores con altas tasas de inmunomarcacion de $\mathrm{Ki}-67$, junto con un mayor riesgo de recurrencia. Los autores relacionan el índice de proliferación celular y las alteraciones en microsatélites, con el pronóstico en ameloblastomas ${ }^{(7)}$. Kuroyanagi et al., sugirió que la expresión de Ki-67 en TOQ en el momento del diagnóstico podría ser útil como marcador pronóstico complementario a procedimientos quirúrgicos para evitar recurrencia. Según los autores, en sujetos con altos índices de Ki-67, podría complementarse con el uso de soluciones de aplicación intraóseas posterior a la enucleación del tumor, dado su alto potencial de recurrencias ${ }^{(16)}$. Sandra et al., el año 2001, realizaron un estudio en el que establecen una correlación entre el índice de Ki-67 y PCNA y la edad, encontrando porcentajes de marcación mayores en la medida que los sujetos envejecían ${ }^{(28)}$. Este aumento en las tasas de proliferación celular relacionadas con el envejecimiento, podría relacionarse con la mayor frecuencia de enfermedades neoplásicas que se presentan en edades avanzadas.

Amol et. al, evaluaron la co-expresión de Ki-67 y p53 en ameloblastoma, TOQ, quiste dentigero (QD), mucosa oral normal
(MON) y mucosa oral fetal (MOF). Sus resultados indican que tanto Ki67 como p53 fueron significativamente mayores en ameloblastoma y TOQ comparados con QD, MON y MOF. Se observó inmunomarcación de Ki-67 y p53, en orden descendente, en ameloblastoma, TOQ, MOF, MON y QD. Los autores sugieren que la expresión de la proteína p53 no implicaría necesariamente una asociación con enfermedad maligna y/o mutación del gen p53, pero habría una tendencia a ser mas expresada, cuantitativa y cualitativamente, en quistes o tumores que tienden a ser más agresivos. Además, señalan que la sobreexpresión de p53 puede promover la proliferación celular en tumores odontogénicos y sugieren que la expresión de Ki-67 y p53 se pueden utilizar como marcadores pronóstico en tumores odontogénicos ${ }^{(29)}$. La p53 utilizada en este estudio, marca tanto el tipo mutada como el tipo silvestre, aunque se sabe que esta última tiene una vida media corta. Si bien no podemos distinguir a que tipo de proteína corresponde la inmunomarcación en nuestro trabajo, si podemos afirmar que fue mayor para p53 en comparación con Ki-67, por lo que su aumento no estaría determinado, al menos de forma exclusiva, por el aumento de la proliferación celular. Aunque la inmunomarcación de p53 fue mayor en ambos tumores estudiados en comparación al control utilizado, debemos considerar que son neoplasias distintas y no necesariamente comparables.

Respecto de p53, Gadbail AR et al. ${ }^{(29)}$ sugiere que una mayor expresión de esta proteína se asocia a un comportamiento biológico más agresivo en tumores odontogénicos y que su expresión puede promover la proliferación celular en las lesiones odontogénicas. Por lo tanto, podrían tomarse medidas terapéuticas similares a las descritas en Ki-67 en la medida que la inmunomarcación de p53 sea mayor en tumores odontogénicos.

Es importante destacar que el conteo celular está sujeto a la interpretación subjetiva que hace el observador, siendo este fenómeno más crítico en los casos en que la tinción de la inmunomarcación es más débil. La mayoría de los estudios no especifíca con escala estandarizada el valor o tono mínimo que se acepta como positivo para la tinción de inmunomarcación, lo que otorga un margen de subjetividad en resultados de distintos autores. De todas formas, los resultados obtenidos sugieren que la inhibición de apoptosis predominó en ambos tumores por sobre la multiplicación celular. Estas diferencias podrían tener relación con el comportamiento biológico localmente agresivo, más cercano a una neoplasia maligna que a una benigna. Dado que AM es un tumor mas agresivo que el TOQ y que por cierto, requiere un tratamiento mucho mas invasivo y radical, podríamos considerar que existen además, otras moléculas no evaluadas en este estudio, que podrían tener injerencia en esta diferencia de comportamiento clínico que se observa entre ambas neoplasias. Se sugiere en lo próximo, junto con aumentar el número muestral para obtener resultados mas representativos, complementar el estudio de estos marcadores, con la detección y relación con elementos de la matriz extracelular. Sería de gran utilidad, detectar y cuantificar proteínas, como las metaloproteinasas de la matriz extracecelular y asociar su presencia con el pronóstico, recurrencia, recidiva y sobrevida de los pacientes. De todas formas, los resultados obtenidos, pueden colaborar en la evaluación del pronóstico y tratamiento de estas neoplasias benignas, incidiendo en definitiva en el tratamiento y calidad de vida de los pacientes.

\section{CONFLICTO DE INTERESES}

Los autores declaran no presentar conflictos de interés de ningún tipo.

\section{REFERENCIAS BIBLIOGRÁFICAS}

1. Avelar RL, Antunes AA, Santos T de S, Andrade ES, Dourado E. Odontogenic tumors: Clinical and pathology study of 238 cases. Braz J Otorhinolaryngol, 2008 Sep-Oct; 74(5): 668-673.

2. Luo HY, Li TJ. Odontogenic tumors: A study of 1309 cases in a chinese population. Oral Oncol, 2009 Aug; 45(8): 706-711.

3. Khalifa GA, Shokier HM, Abo-Hager EA. Evaluation of neoplastic nature of keratocystic odontogenic tumor versus ameloblastoma. J Egypt Natl Canc Inst, 2010 Mar; 22(1): 61-72.

4. Meer S, Galpin JS, Altini M, Coleman $\mathrm{H}$, Ali H. Proliferating cell nuclear antigen and Ki-67 immunoreactivity in ameloblastomas. Oral Surg Oral Med Oral Pathol Oral Radiol Endod, 2003 Feb; 95(2): 213-221.

5. Mendes RA, Carvalho JF, Van der Waal I. Biological pathways involved in the aggressive behavior of the keratocystic odontogenic tumor and possible implications for molecular oriented treatment - An overview. Oral Oncol, 2010 Jan; 46(1): 19-24.
6. Mendes RA, Carvalho JF, Van der Waal I. A comparative immunohistochemical analysis of COX-2, p53, and $\mathrm{Ki}-67$ expression in keratocystic odontogenic tumors. Oral Surg Oral Med Oral Pathol Oral Radiol Endod, 2011 Mar; 111(3): 333-339.

7. Migaldi M, Sartori G, Rossi G, Cittadini A, Sgambato A. Tumor cell proliferation and microsatellite alterations in human ameloblastoma. Oral Oncol, 2008 Jan; 44(1): 50-60. 8. Florescu A, Simionescu C, Ciurea R, Pitru A. p53, Bcl-2, and Ki-67 inmmunoexpression in follicular solid ameloblastomas. Rom J Morphol Embryol, 2012; 53(1): 105-109.

9. Lothaire P, de Azambuja E, Dequanter D, Lalami Y, Sotiriou C, Andry G Castro G Jr, Awada A. Molecular markers of head and neck squamous cell carcinoma: Promising signs in need of prospective evaluation. Head Neck 2006 Mar; 28(3): 256-269.

10. Roa JC, Roa I, Melo A, Araya A, Villaseca H, Flores M, Schneider B. Mutación del gen p53 en el cáncer de colon y recto. Rev Méd Chile, 2000; 128(9): 996-1004. 
11. Finlay CA, Hinds PW, Levine AJ. The p53 proto-oncogene can act as a suppressor of transformation. Cell, 1989 Jun 30; 57(7): 1083-1093.

12. Kumamoto $\mathrm{H}$, Izutsu T, Ohki K, Takahashi N, Ooya K. p53 gene status and expression of p53, MDM2, and p14 proteins in ameloblastomas. J Oral Pathol Med, 2004 May; 33(5): 292-299.

13. Montebugnoli L, Badiali G, Marchetti C, Cervellati F, Farnedi A, Foschini MP. Prognostic value of Ki-67 from clinically and histologically 'normal' distant mucosa in patients surgically treated for oral squamous cell carcinoma: A prospective study. Int J Oral Maxillofac Surg, 2009 Nov; 38(11): 1165-1172.

14. Lee JI, Jin BH, Kim MA, Yoon HJ, Hong SD. Prognostic significance of CXCR-4 expression in oral squamous cell carcinoma. Oral Surg Oral Med Oral Pathol Oral Radiol Endod, 2009 May; 107(5): 678-684.

15. Wang Z, Zhang B, Jiang L, Zeng X, Chen Y, Feng X, Guo Y, Chen Q. RACK1, an excellent predictor for poor clinical outcome in oral squamous carcinoma, similar to Ki-67. Eur J Cancer, 2009 Feb; 45(3): 490-496.

16. Kuroyanagi N, Sakuma H, Miyabe S, Machida J, Kaetsu A, Yokoi M et al. Prognostic factors for keratocystic odontogenic tumor (Odontogenic keratocyst): Analysis of clinico-pathologic and immunohistochemical findings in cysts treated by enucleation. J Oral Pathol Med, 2009 Apr; 38(4): 386-392.

17. Philipsen HP, Reichar PA, Slootweg PJ, Slater LJ. Neoplasms and tumourlike lesions arising from the odontogenic apparatus and maxillofacial skeleton: Introduction. Pathology and genetics of head and neck tumours. WHO. IARC Press Lyon, 2005; 6: 285-328.

18. de-Vicente JC, Torre-Iturraspe A, Gutiérrez AM, Lequerica-Fernández $P$ Immunohistochemical comparative study of the odontogenic keratocysts and other odontogenic lesions. Med Oral Patol Oral Cir Bucal, 2010 Sep 1; 15(5): 709-715.

19. Barboza CA, Pereira Pinto L, Freitas Rde A, Costa Ade L, Souza LB. Proliferating cell nuclear antigen (PCNA) and p53 protein expression in ameloblastoma and adenomatoid odontogenic tumor. Braz Dent J, 2005; 16(1): 56-61.
20. Amaral FR, Mateus GC, Bonisson LA, de Andrade BA, Mesquita RA, Horta MC, Marigo Hde A. Cell proliferation and apoptosis in ameloblastomas and keratocystic odontogenic tumors. Braz Dent J, 2012; 23(2): 91-96.

21. Salehinejad J, Zare-Mahmoodabadi R, Saghafi S, Jafarian AH, Ghazi N, Rajaei AR. Immunohistochemical detection of p53 and PCNA in ameloblastoma and adenomatoid odontogenic tumor. J Oral Sci, 2011 Jun; 53(2): 213-217.

22. Piattelli A, Fioroni M, Santinelli A, Rubini C. p53 protein expression in odontogenic cysts. J Endod, 2001 Jul; 27(7): 459-461.

23. Shear $M$. The aggressive nature of the odontogenic keratocyst: Is it a benign cystic neoplasm? Part 2: Proliferation and genetic studies. Oral Oncol, 2002 Jun 38(4): 323-331.

24. Slootweg PJ. p53 protein and Ki-67 reactivity in epithelial odontogenic lesions An immunohistochemical study. J Oral Pathol Med, 1995 Oct; 24(9): 393-397.

25. González-Moles MA, Mosqueda-Taylor A, Delgado-Rodríguez M, MartínezMata G, Gil-Montoya JA, Díaz-Franco MA et al. Analysis of p53 protein by PAb240, $\mathrm{Ki}-67$ expression and human papilloma virus DNA detection in different types of odontogenic keratocyst. Anticancer Res, 2006 Jan-Feb; 26(1A): 175-181.

26. Li TJ, Browne RM, Prime SS, Paterson IC, Matthews JB. p53 expression in odontogenic keratocyst epithelium. J Oral Pathol Med, 1996 May; 25(5): 245-255.

27. Soluk Tekkeşın M, Mutlu S, Olgaç V. Expressions of bax, bcl-2 and Ki-67 in odontogenic keratocysts (keratocystic odontogenic tumor) in comparison with ameloblastomas and radicular cysts. Turk Patoloji Derg, 2012; 28(1): 49-55.

28. Sandra F, Mitsuyasu T, Nakamura N, Shiratsuchi $Y$, Ohishi M Immunohistochemical evaluation of PCNA and Ki-67 in ameloblastoma. Oral Oncol, 2001 Feb; 37(2): 193-198.

29. Gadbail AR, Patil R, Chaudhary M. Co-expression of Ki-67 and p53 protein in ameloblastoma and keratocystic odontogenic tumor. Acta Odontol Scand, 2012 Dec; 70(6): 529-535. 